\title{
La legislación de la Provincia de Santa Fe frente a las asimetrías regionales de desarrollo económico, 1991-2019
}

\author{
Legislation of the Province of Santa Fe and Regional Asymmetry \\ in Economic Development, 1991-2019
}

Mauricio Moltó Morresi*

\section{RESUMEN}

La Provincia de Santa Fe es una de las regiones con mayor desarrollo relativo de Argentina. Sin embargo, existen notorias asimetrías económicas internas si comparamos sus distintas regiones geográficas, las cuales cristalizaron en el sentido común en términos de la distinción entre un norte pobre y despoblado y un sur con mayor nivel de desarrollo y población. En consideración de esto, los objetivos propuestos son: observar la distribución geográfica de las iniciativas relacionadas con el desarrollo económico instrumentadas desde la provincia, analizar aquellas propuestas que contribuyeron a achicar la brecha norte-sur y describir continuidades y diferencias al interior del periodo 1991-2019. Como resultado, se vio que el Estado santafesino dirigió mayormente su atención hacia las regiones más consolidadas de la Provincia y no pudo articular iniciativas a largo plazo para achicar la brecha norte-sur.

\section{ABSTRACT}

The Province of Santa Fe is one of the regions with the highest level of development in Argentina. However, there are distinct internal economic asymmetries when we compare its different
Palabras

clave: Estados

provinciales,

asimetrías

regionales,

desarrollo

regional, Provincia

de Santa Fe.

Keywords

Provincial

states, regional

Argentino. Doctor en Ciencias Sociales, Universidad de Buenos Aires, Argentina. Becario posdoctoral CONICET en el Instituto de Humanidades y Ciencias Sociales de CONICET y la Universidad Nacional del Litoral (IHuCSo- CONICET- UNL). E-mail: mauriciomolto@gmail.com

Este trabajo es parte de una investigación mayor titulada "Iniciativas provinciales para la promoción del desarrollo económico. Santa Fe 1991-2015”, presentada para obtener el grado académico de Magíster en Desarrollo y Gestión Territorial, Facultad de Ciencias Económicas y Estadísticas, Universidad Nacional de Rosario, Argentina. 
geographic regions, which were commonly translated in terms of the distinction between a poor, unpopulated north sector and a more populated and developed south sector. In this sense, the aims of this article are to observe the geographic distribution of initiatives related to economic development organized by the province, to analyze those proposals that helped bridge the north-south gap, and to describe continuities and differences within the period between 1991 and 2019. As a result, we see how the Santa Fe government directed most of its attention to the most consolidated regions of the Province and was unable to carry out long-term initiatives to close the north-south gap. asymmetries, regional development, Province of Santa Fe. 
Durante el último cuarto del siglo XX, el Estado nación vio relativizada su posición de núcleo articulador de la sociedad e, incluso, la propia idea de sociedad dejó de estar asociada al ámbito nacional. La erosión de la centralidad del Estado se dio tanto por arriba como por abajo, ya que ganaron mayor protagonismo instituciones y movimientos culturales e identitarios globales y locales, quedando en discusión el rol jugado por el propio Estado en este proceso (Vilas, 2005).

En este periodo, la teoría económica neoliberal desplazó al keynesianismo y se convirtió en la nueva ortodoxia, y, a la vez que articuló fuertes críticas hacia los modelos de planificación centralizados, puso el acento en la "competitividad" de los territorios y su "crecimiento endógeno". Esto último dio paso a una situación que estimuló la competencia entre regiones bajo el imperativo de crear un ambiente atractivo que permitiera aumentar el potencial para captar inversiones externas en capital físico, humano y/ tecnológico (De Mattos, 2012).

Esta situación ocasionó la emergencia de regiones ganadoras y perdedoras en el nuevo orden global y, en algunos casos, acrecentó las diferencias al interior de los propios Estados nacionales. En este marco, las provincias se encontraron haciendo frente a los imperativos de incrementar la competitividad territorial, coadyuvar la innovación en el sector privado y trabajar en estrategias innovadoras de gestión, generar sinergias, construir redes de cooperación con y entre los actores sociales locales, y trabajar en la inserción de esas redes en los circuitos globales.

Argentina es un Estado federal, organizado en veintitrés provincias y la Ciudad Autónoma de Buenos Aires. De acuerdo con la Constitución Nacional, los Estados provinciales mantienen todas las atribuciones no delegadas al Estado nacional. Esto implica que gozan de cierta autonomía para la instrumentación de políticas públicas sobre un amplio espectro de temas. En años recientes, las ciencias sociales se han dedicado al estudio de las dinámicas sociales, económicas y políticas de los Estado provinciales (Barros, Castellani y Gantus, 2016; Mauro, Ortiz de Rozas y Vaca Narvaja, 2016; Ortiz de Rozas, 2016), dando forma a un fecundo campo de estudios en las ciencias sociales en Argentina.

La Provincia de Santa Fe posee una economía de primera importancia en Argentina. No puede decirse que sea una región ganadora en el nuevo escenario global, ya que posee una economía basada en la pro- 
ducción e industrialización de materias primas y una estructura productiva en la que predominan pequeñas y medianas industrias intensivas en mano de obra y de baja capacidad tecnológica; pero, de todos modos, es una de las regiones con mayor desarrollo relativo del país. Sin embargo, existen notorias asimetrías internas, las cuales cristalizaron en el sentido común en términos de la distinción entre un norte pobre y despoblado y un sur con mayor nivel de desarrollo y población.

El origen de esta asimetría puede encontrarse en el proceso de expansión que experimentó la economía provincial durante la segunda mitad del siglo XIX y principios del XX, ligado a la producción de bienes agropecuarios, en consonancia con la inserción internacional de Argentina en el mercado mundial de entonces; consecuencia de lo cual, el sur santafesino, con Rosario como núcleo articulador, fue consolidándose como la zona más dinámica, a la vez que se transformó en el área con mayor concentración de población. Este periodo sentó las bases de una desigualdad que llega hasta nuestros días y es reconocida por el propio Estado de Santa Fe como una deuda histórica.

En consideración de esta situación, los objetivos propuestos son observar la distribución geográfica de las iniciativas relacionadas con el desarrollo económico instrumentadas desde la provincia, analizar aquellas propuestas que contribuyeron a achicar la brecha norte-sur y establecer continuidades y diferencias al interior del periodo 1991-2019.

\section{Marco de análisis}

Se parte de la idea de que "la vida económica es un proceso instituido como una actividad socialmente enraizada" (Amin, 1998: 71). Esto implica que el mercado no es el punto de partida natural de la actividad económica, sino que se trata de instituciones históricas, construidas socialmente sobre la base de intereses contrapuestos, de agentes dotados con volúmenes de recursos diferentes que les otorgan mayor o menor capacidad para imponer las reglas de juego (Beckert, 2009; Granovetter, 1998; Polanyi, 2007). También implica reconocer que los individuos son parte de un entramado social mayor, en el que su acción se encuentra enraizada (Granovetter, 1973). Esta mirada contrasta fuertemente con la ortodoxia neoliberal, según la cual el mercado es el mecanismo óptimo y natural de la vida económica, que regula la interacción de individuos racionales motivados por la maximización de beneficios. 
Otro punto de partida es una idea de "desarrollo económico" que hace hincapié en la necesidad de un "cambio estructural de los patrones de producción, el avance tecnológico y una modernización social, política e institucional", que, a su vez, considera el impacto sobre el medioambiente y el buen desempeño institucional (Fanelli y Popov, 2003, citado en Orlansky, 2005: 45 y 46).

De este modo, ya se trate de una descripción, un diagnóstico o el diseño de una estrategia para lograr el desarrollo económico de un territorio en particular, es necesario considerar a los agentes económicos como parte de un entramado relacional en el que, si bien juegan las motivaciones económicas, hay una estructura de reglas y recursos institucionalizados y no institucionalizados que exceden el plano de la economía, e impactan directamente sobre la actividad económica y su posibilidad de desarrollo.

En este sentido, resulta útil considerar también los aportes realizados desde la perspectiva de desarrollo territorial. Este enfoque se basa en la idea de que los actores locales pueden articular estrategias para un crecimiento cuantitativo y cualitativo de la actividad económica y las condiciones de vida de los habitantes sobre la base de los recursos disponibles en el propio territorio, que no necesariamente remite a una sola localidad, sino que puede abarcar varios municipios o una región con características similares. Además, supone un análisis de las capacidades de los territorios que se diferencia de los análisis sectoriales (Albuquerque y Pérez Rozzi, 2013; Albuquerque, 2004; Boiser, 2001; Vázquez Barquero, 2000).

En este marco, con el fin de promover el desarrollo territorial, son claves las capacidades del Estado para coordinar de forma centralizada las actividades de la sociedad civil (Mann, 2006) y su enraizamiento (Evans, 1996) en el territorio. Que en este caso se trate de un Estado provincial impacta sobre su posibilidad de acción autónoma frente al Estado central, pero, de todos modos, sin perder de vista esta diferencia, la perspectiva sobre capacidades estatales resulta interesante para articular el análisis.

La idea de "capacidades estatales" refiere a la posibilidad de un Estado de trazar objetivos propios y llevar adelante acciones para dar cumplimiento de ellos de forma eficiente. 
Como parte de las capacidades de un Estado, Repetto (2004) señala que las capacidades políticas pueden ser entendidas como la posibilidad de los dirigentes de identificar, analizar y dar respuestas a las demandas de la sociedad, los vínculos establecidos con el entorno social y económico y las reglas de juego que regulan las relaciones entre ellos. En esta línea, Alonso (2007: 15) propone un "análisis relacional de las capacidades estatales", en el sentido de "inducir comportamientos entre los actores no estatales, logrando el cumplimiento obligatorio de las reglas de juego y evitando los bloqueos a sus políticas y entendiendo a las instituciones como el logro de equilibrios que reflejan (...) el modo en que se dirimen conflictos entre intereses sociales contrapuestos". Bertranou (2013), por su parte, también destaca la importancia de las vinculaciones con los actores no estatales y el "capital de acción interinstitucional", entendido como el acervo de relaciones con otras organizaciones y la posibilidad de acción conjunta. A su vez, Fernández y Vigil (2012 y 2010) destacan los mecanismos que contemplan la participación de los actores territoriales en el proceso de armado y ejecución de una política pública, la evaluación que los actores implicados realizan de la iniciativa y de las capacidades del Estado para su desarrollo, la propia capacidad organizacional de estos actores, los resultados esperados y la incidencia que pretenden tener en su ejecución.

De este modo, entonces, se trata de analizar las iniciativas para el desarrollo económico instrumentadas por el Estado provincial en el territorio santafesino, con base en la idea de desarrollo territorial y sus capacidades para llevar adelante sus propuestas de acuerdo con una lógica colaborativa y en miras a un acoplamiento exitoso con lógicas y dinámicas de mayor escala.

\section{Estrategia metodológica}

Se trabajó con las leyes sancionadas por la Legislatura Provincial entre 1991 y 2019, relacionadas con cuestiones de desarrollo económico, con el fin de observar y describir los vínculos que establece la legislación de la Provincia de Santa Fe con las diferentes áreas geográficas de su territorio y los actores locales. Se trabajó con una base de datos confeccionada para la escritura de un trabajo tesis de maestría en 2016, que para el presente artículo fue actualizada hasta 2019. El periodo tempo- 
ral elegido permite comparar el comportamiento del Estado provincial en materia económico-productiva durante distintos subperiodos que, desde el punto de vista macroeconómico, tienen diferencias bien marcadas. De este modo, se optó también por hacer coincidir el comienzo y el final de la serie con los inicios de los gobiernos provinciales. Así, el intervalo temporal va desde el 10 de diciembre de 1991 al 10 de diciembre de 2019. A principios de la década de los 90 y hasta diciembre de 2001, los lineamientos macroeconómicos en Argentina estuvieron en línea con la apertura y la desregulación de la economía, en la que se privilegiaron iniciativas promercado que avanzaron en la desregulación de la economía y la transferencia de empresas públicas al sector privado. Estos lineamientos cambiaron luego de la crisis económica, política y social de diciembre de 2001, dando paso a lineamientos macroeconómicos en los que el Estado nacional desplegó acciones tendientes a una mayor regulación e intervención sobre la esfera económica entre 2003 y 2015, aunque, como se hará más adelante en el cuerpo del artículo, pueden reconocerse distintas etapas al interior de este intervalo temporal. Luego de 2015 y hasta 2019, los lineamientos macroeconómicos viraron nuevamente hacia formas que privilegiaron la acción de grandes actores económicos y la desregulación de la economía, principalmente en lo que respecta a los servicios públicos, el sistema financiero y el comercio exterior. De esta forma, se pueden observar las respuestas desde el Estado provincial a las distintas coyunturas macroeconómicas y, a su vez, ver si existen diferencias entre las distintas gobernaciones que se sucedieron cada cuatro años.

Se presentará un panorama general acerca de las vinculaciones del Estado de Santa Fe con las distintas áreas de la provincia, que dará una idea sobre el direccionamiento territorial de políticas públicas para la promoción del desarrollo económico en un intervalo temporal relativamente largo, aunque no permitirá realizar una valoración sobre el impacto de estas iniciativas, debido a las limitaciones de información sobre la implementación de las políticas mencionadas.

Los vínculos que se cuantifican y se describen en las siguientes páginas funcionan como un indicador de la acción territorial del Estado provincial. Se construyeron identificando ciudades, departamentos y áreas geográficas mencionadas en las leyes sancionadas por la Legislatura Provincial entre 1991 y 2019, con referencia a cuestiones de desarrollo eco- 
nómico. El recorte temporal permitió avanzar en una comparación entre los años 90 y la posconvertibilidad. Por otro lado, la serie inicia y termina en fechas de inicio de nuevos mandatos provinciales, lo cual permite incluir siete periodos de gobierno y ver también diferencias entre ellos.

Se eligió trabajar con este tipo de documentos, porque permitieron una reconstrucción sistemática de la situación en un periodo temporal largo; aunque existe conciencia de las limitaciones que esto representa. Por un lado, debe tenerse en cuenta que las iniciativas contempladas en la legislación no agotan el abanico de acciones desarrolladas por el Estado provincial en el periodo analizado; además que, en paralelo, funciona un entramado informal de relaciones que permanece oculto e impacta sobre la realidad económica local. Por otro lado, debe considerarse que en el proceso de aplicación y puesta en marcha de una ley operan factores que pueden ocasionar una distancia entre la norma, su aplicación y los resultados esperados. Por estos motivos, la información presentada es representativa de acciones potenciales (antes que de hechos concretos) establecidas a través de uno de los canales formales existentes entre el Estado provincial y su territorio. Además, el contenido de los documentos es, en la mayoría de los casos, escueto y no brinda detalles sobre las líneas de acción seguidas.

El trabajo no trata sobre el desarrollo económico en sí mismo, sino sobre el direccionamiento territorial de las iniciativas provinciales para su promoción en distintas áreas geográficas. De todos modos, el concepto de "desarrollo económico" fue utilizado para la selección de los documentos analizados y se operacionalizó con base en leyes del Estado provincial que avanzaron en la línea de: 1) promoción y apoyo a sectores tecnológicos y difusión de tecnología; 2) iniciativas destinadas a la planificación, activación o fortalecimiento de la economía de áreas específicas de la provincia, tanto en lo que toca a infraestructura como respecto al apoyo a sectores específicoslocalizados;3)el "reconocimiento simbólico" o declaraciones de interés o de estatus sobre una actividad, localidad o región asociada a un producto o área de la producción determinada; 4) incentivos fiscales, subsidios y créditos; 5) legislación relacionada con la regulación del uso, adecuación o construcción de obras públicas necesarias para un mejor desenvolvimiento de la producción; 6) iniciativas que apuntan al beneficio o la regulación de la producción de sectores específicos; 7) creación de instituciones 
como entes, comisiones, programas especiales, planes estratégicos, sistemas de información, empresas públicas y mixtas, observatorios, etc., tendientes a facilitar la acción, coadyuvar o desarrollar áreas específicas de la producción; 8) toma de deuda y devolución por parte de la provincia para la construcción de infraestructura o la autorización a contraer deuda a municipios y comunas para los mismos fines; 9) leyes de emergencia económica sobre empresas recuperadas y retribución por acción de hechos extraordinarios como desastres climáticos; 10) regulaciones productivas tendientes a la disminución del impacto ecológico de la producción, y 11) normativa relacionada con la actividad de sectores económicos específicos.

Se accedió a las leyes analizadas por medio del Sistema de Información Normativa de la Provincia de Santa Fe. El análisis de la información se realizó con el software Atlas.ti. ${ }^{\circ}$ Con el software se realizó el análisis de los documentos y se identificó las categorías con las cuales, luego, se confeccionó una base de datos cuantitativa, cuyos resultados se volcaron en las tablas que se presentan en el cuerpo de este y otros dos trabajos previos realizados con la misma estrategia metodológica.

\section{Alcance geográfico de las iniciativas}

Entre el 10 de diciembre de 1991 y esa misma fecha de 2019, el Estado provincial sancionó 399 leyes relacionadas con cuestiones de desarrollo económico. Entre los documentos, se encontró que un número importante de ellas hace referencia a áreas o localidades específicas. En ese sentido, el análisis de las menciones permitió ver qué zonas fueron mayormente contempladas (Tabla $\mathrm{N}^{\circ} 1$ ).

Las referencias geográficas se encontraron en cuatro escalas: 1) provincial; 2) regional, como "la costa" o "el noroeste"; 3) departamental y 4) local. Las menciones no son mutuamente excluyentes y en muchos de los documentos se encuentran referencias a más de una localidad o departamento.

En la Provincia de Santa Fe existen 51 municipios y 312 comunas. Son municipios aquellos agrupamientos poblacionales con más de 10 mil habitantes, los cuales, a su vez, son clasificados como de primera categoría si superan los 200 mil habitantes, o de segunda aquellos con 
una cifra entre 10 mil y 200 mil. Solo Rosario y Santa Fe son de primera categoría. A su vez, el territorio está dividido en 19 departamentos que son, básicamente, unidades administrativas; pero poseen cierta relevancia política debido a que cada uno tiene un representante en la Cámara de Senadores provincial.

El Producto Bruto Geográfico (PBG) de Santa Fe equivale al 8\% del Producto Bruto Interno (PBI) argentino y el $24 \%$ de las exportaciones argentinas. Aproximadamente el $40 \%$ del PBG se explica por sectores productores de bienes, entre los que destacan la industria manufacturera $(23,7 \%)$ y el sector agropecuario $(13,6 \%)$. Entre las exportaciones destacan las manufacturas de origen agropecuario y de origen industrial, las que representan el $64,5 \%$ y el $22,4 \%$ de lo exportado por la provincia.

La industria manufacturera es la que más contribuye al PBG $(23,7 \%)$ y da empleo al $26,3 \%$ de la población económicamente activa (empleo registrado). En orden de importancia siguen los servicios inmobiliarios, empresariales y de alquiler, con una participación del 20,6\% en el PBG, pero responsable del 9,7\% del empleo formal provincial; el sector agropecuario, 13,6\% del PBG y 5,3\% del empleo; y el comercio al por mayor, al por menor y reparaciones, 10,7\% del PBG y 19,3\% del empleo. Estos datos muestran la importancia de los sectores manufacturero y agropecuario en la producción de valor, que explican el 37,6\% del PBG.

En lo que toca a las exportaciones, tienen un peso relativo mayor las manufacturas de origen agropecuario (MOA) (64,5\%). Le siguen en importancia las manufacturas de origen industrial (MOI) (22,4\%) y los productos primarios (12,3\%). Las exportaciones de combustibles y energía representan solo el $0,8 \%$. La producción de mayor peso es el aceite de soja y sus derivados, que explican el $\mathbf{8 3 \%}$ de las MOA.

Las actividades primarias más relevantes son el cultivo de soja, maíz, trigo, girasol, sorgo y algodón, y la producción de leche y de ganado bovino. Estos productos dan lugar a actividades agroindustriales que tienen una importancia de primer orden: aceites y derivados, molienda de trigo, faena de bovinos y productos lácteos. El cultivo e industrialización de soja, maíz y trigo predomina en el centro y sur de la provincia. El girasol, el sorgo y el algodón tienen mayores volúmenes de producción en los departamentos del norte, pero la industrialización de este último se da, mayormente, en el sur. La producción de leche y su industrialización es característica del centro de la provincia, 
aunque también existen volúmenes significativos de producción en el sur (Acosta, 2011b; 2011d; 2012b; y Donato, 2010).

Por otro lado, las manufacturas de origen industrial más relevantes se encuentran en las cadenas de producción de la maquinaria agrícola, de maquinarias para la industria alimenticia, de la industria autopartista, en la fabricación de carrocerías y remolques, y la de madera y muebles. La producción de maquinaria agrícola predomina en el suroeste, más específicamente en los departamentos Belgrano, Caseros y General López, aunque también es significativa en Rosario y Castellanos. La fabricación de bienes de capital para la industria alimenticia es importante en los departamentos del centrosur de la provincia; al igual que las actividades vinculadas con la fabricación de carrocerías y remolques. Las empresas vinculadas con la industria autopartista predominan en la zona del Gran Rosario y en los departamentos del centro y sur. La cadena de madera y muebles destaca en Iriondo y Las Colonias (Acosta, 2011a; 2011c; 2011e; 2011f; 2012a; y Donato, 2010). Por otro lado, en Constitución existe un importante desarrollo de la industria siderúrgica, principalmente en la ciudad de Villa Constitución, y en San Lorenzo destaca también la actividad petroquímica (ambas en la zona sureste).

A los fines de ordenar la información, se agregaron las referencias a localidades y departamentos en siete áreas:

1) Cordón industrial: incluye las referencias a la franja costera de los departamentos San Lorenzo, Rosario y Constitución, donde gravita muy fuerte el Gran Rosario, de aproximadamente 1,2 millones de habitantes (INDEC, 2010) e importante presencia de industria alimenticia (principalmente aceites y derivados), automotriz, cárnica, metalúrgica, siderúrgica, petroquímica, calzado y de software y servicios informáticos.

2) Área pampeana: incluye todo el sur de Santa Fe hasta el límite que forma el inicio de los departamentos San Martín y San Jerónimo, y excluye la zona delimitada en el punto 1, aproximadamente de 450 mil habitantes (INDEC, 2010). Destaca la producción de soja, maíz y trigo; industria metalúrgica (principalmente maquinaria agrícola), carrocerías y remolques, frigorífica, de madera y muebles y láctea.

3) Gran Santa Fe: incluye las ciudades de Santa Fe (capital provincial), Santo Tomé, Recreo y San José del Rincón, y las comunas de Sauce 
Viejo, Arroyo Leyes y Monte Vera. Aproximadamente de 530 mil habitantes (INDEC, 2010), destacan la industria alimenticia (producción de cervezas principalmente) y metalúrgica.

4) Cuenca lechera: los departamentos San Martín, San Jerónimo (excepto la franja costera), Las Colonias, Castellanos, San Justo y el sur de San Cristóbal. Aproximadamente de 370 mil habitantes (INDEC, 2010), produce ganado bovino, de soja y maíz; industria láctea, autopartes, bienes para la industria alimenticia, cárnica, metalúrgica y madera y muebles.

5) Noreste: se agruparon las menciones a los departamentos General Obligado y a la ciudad de Vera. Aproximadamente de 230 habitantes (INDEC, 2010), produce algodón, madera, girasol, soja, sorgo, caña de azúcar, ganado bovino y cueros; industria de hilados y madera y muebles.

6) La costa (denominación tomada de la legislación): incluye a Garay, San Javier y el este de San Jerónimo. Aproximadamente de 80 mil habitantes (INDEC, 2010), sus actividades productivas principales son la pesca y el cultivo de frutas y hortalizas.

7) Noroeste (denominación también tomada de los documentos analizados): incluye parte de Vera, parte de San Cristóbal y el departamento Nueve de Julio. Aproximadamente de 60 mil habitantes (INDEC, 2010), produce ganado bovino, madera, algodón y oleaginosas.

Tabla $\mathrm{N}^{\circ} 1$

Menciones a localidades, departamentos y regiones en las leyes sancionadas por la Legislatura agregadas por áreas. Provincia de Santa Fe, 1991- 2019

\begin{tabular}{lc}
\hline \multicolumn{1}{c}{ Área geográfica } & Menciones \\
\hline Cordón industrial & 42 \\
\hline Gran Rosario & 30 \\
\hline Gran Santa Fe & 25 \\
\hline Cuenca Lechera & 23 \\
\hline Área pampeana & 20 \\
\hline Noreste provincial & 18 \\
\hline Reconquista- Avellaneda & 8 \\
\hline La Costa & 11 \\
\hline Noroeste Provincial & 2 \\
\hline Total & 143 \\
\hline
\end{tabular}

Fuente: Elaboración propia. 
En la Figura $\mathrm{N}^{\circ} 1$, se muestra una delimitación aproximada de las áreas geográficas mencionadas:

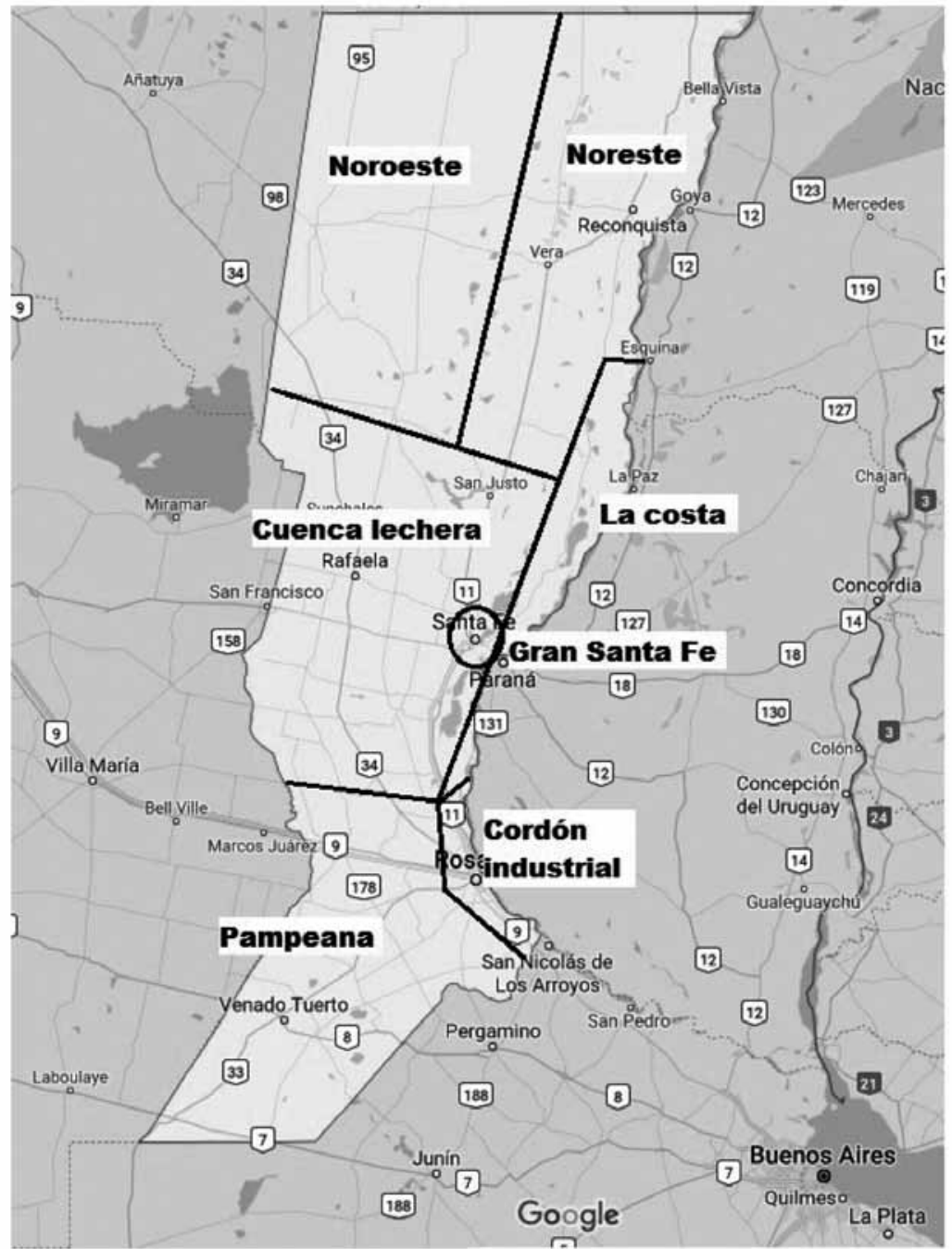

Figura $N^{\circ} 1$. Clasificación áreas de la provincia de Santa Fe Fuente: Elaboración propia en base a Google Maps. 


\section{Descripción de las iniciativas por área}

\section{Gran Rosario y cordón industrial}

Entre las iniciativas destinadas al área del sudeste santafesino, conocida tradicionalmente como "cordón industrial", predominan aquellas dirigidas hacia el Gran Rosario. Una de las líneas más importantes, que presenta continuidad a lo largo del periodo, es el establecimiento de parques o áreas destinadas a la actividad industrial. En este sentido, la normativa habilitó la cesión de terrenos para la creación de parques y/o áreas industriales en Funes (1993), Puerto General San Martín (1995), Fray Luis Beltrán (1998), San Lorenzo (2001), Roldán (2005) y Pérez (2007). También, se declaró como zona para el desarrollo industrial a los distritos de Alvear, Pueblo Esther, General Lagos y Villa Amelia, en el límite sur y suroeste del aglomerado (1995).

Fueron importantes las iniciativas portuarias. Durante los años 90 se transfirió, de forma gratuita, la administración, explotación y dominio a la órbita provincial por parte de Nación, y a los municipios, por parte de la Provincia, de varios puertos de la franja costera del Río Paraná. En el Gran Rosario se creó el Ente Administrador del Puerto de Rosario y se transfirió a la Municipalidad de Puerto General San Martín el puerto de esa localidad (1995). Lo mismo ocurrió en Villa Constitución, a 50 kilómetros de allí, en el extremo sureste de la provincia, donde se creó el Ente Administrador del Puerto de Villa Constitución (1994). Además, se transfirió al municipio de esta ciudad la explotación y administración de las instalaciones de la ex "Junta Nacional del Grano", ubicadas en la zona portuaria de esa ciudad (2005).

También en Villa Constitución se creó la Zona Franca Santafesina. Si bien en 1994 se aprobó un convenio para tal fin con el Estado Nacional, a juzgar por las fechas de la normativa relacionada con este hecho, la iniciativa tuvo un avance lento y hubo que esperar hasta el año 2000 para que se produjeran avances significativos (al menos en la legislación). En ese año se creó, en el Ministerio de Agricultura, Ganadería, Industria y Comercio de la Provincia, el "Ente Zona Franca Santafesina", con funciones como "Comité de vigilancia de la Zona Franca de Villa Constitución". En ese año también se declaran de utilidad pública y sujetos a expropiación los inmuebles que el Ente 
considere necesario para el funcionamiento de la zona franca. Pero la adquisición de los inmuebles y localización no fue habilitada sino hasta 2003.

Por otro lado, se aprobó la creación de un trade point, de forma conjunta entre la Municipalidad de Rosarioy el Ente Administrador del Puerto de esta ciudad, cuyo objetivo es la generación de información, asesoramiento técnico, promoción de negocios internacionales, atención a pequeñas y medianas empresas (PyME) y el desarrollo de misiones de inversión (1997). En el área de comercio exterior, se promovió la participación de PyME mediante un convenio con la fundación Export.Ar en Rosario (1996) y la creación del "Centro de promoción de negocios internacionales", también en esa ciudad (1999).

En Rosario también se creó el "Centro Regional Rosario", años más tarde INTI Rosario, mediante un convenio con la Universidad Nacional de Rosario (UNR), el municipio, el Instituto Nacional de Tecnología Industrial (INTI) y organizaciones de la producción (1999); y un "Centro asesor de empresas", también en conjunto con UNR (1999).

La crisis económica de principios de los años 2000 impactó muy fuerte al área del Gran Rosario. En ese marco, muchos establecimientos industriales y comerciales pasaron a ser administrados de hecho por los trabajadores, conformando cooperativas. En este sentido, desde la provincia se normalizó el funcionamiento de estas empresas y se habilitó la explotación por parte de los trabajadores sobre las instalaciones, maquinarias y herramientas afectadas por esta situación (2004, 2006 y 2009). En 2016 se sancionó una normativa marco para la regulación de las actividades de todas las empresas recuperadas de la provincia.

En materia ambiental, se promovió la reconversión productiva de Celulosa Argentina SA, en la localidad de Capitán Bermúdez, en el norte del Gran Rosario (2009).

Para destacar es la creación del Centro Binacional de Genómica Vegetal, mediante un convenio entre el Consejo Nacional de Investigaciones Científicas y Técnicas (CONICET), UNR, la Municipalidad de Rosario, el Gobierno de la República Argentina y el Reino de España (2006). 
Otras iniciativas están relacionadas con el apoyo a productores minifundistas (1995), bonificaciones de tasas en créditos para la reactivación productiva, otorgados por el Consejo Federal de Inversiones (CFI), en 2007, la promoción para incorporar nuevos trabajadores a la actividad productiva provincial y elevar la productividad y la calidad de las industrias (2007). Por ley, también, se dio estatus de "Capital Provincial del Mutualismo" a Rosario (2006), "del Acero" a Villa Constitución (2007), "del Calzado" a Arroyo Seco (2010) y "de la Industria Frigorífica" a Villa Gobernador Gálvez (2010).

\section{Gran Santa Fe}

El otro polo urbano de relevancia en la provincia lo constituye el área del Gran Santa Fe. Esta zona también recibió una atención relativamente alta por parte de la legislación en el periodo, con algunas similitudes y diferencias con respecto al Gran Rosario.

La cuestión portuaria fue la que mayor atención recibió, contándose seis leyes provinciales en ese sentido. La historia de la ciudad de Santa Fe se encuentra estrechamente ligada a su puerto, pero su desarrollo durante el siglo XX ha corrido una suerte errática en comparación con los puertos del sur de la provincia. Por esa razón, aquí solo se detallan las leyes provinciales, sin entrar en detalle sobre el devenir de las acciones e iniciativas, ya que eso sería un trabajo de investigación en sí mismo.

En 1994, el Estado nacional transfiere el puerto de la Ciudad de Santa Fe a la provincia y se crea el Ente Administrador del Puerto de Santa Fe. Un año más tarde se crea un trade point "destinado a implementar un centro único de concentración de factores relacionados al comercio exterior". En 1998 se transfiere a la Empresa Provincial de la Energía las instalaciones eléctricas del puerto de Santa Fe, con la finalidad de realizar la provisión de energía a los usuarios radicados en esa jurisdicción de forma directa.

Por otro lado, años más tarde, para la reconversión del puerto, se firma un convenio de carácter financiero con el Fondo Financiero para el Desarrollo de los Países de la Cuenca del Plata (FONPLATA), del cual fue garante el Estado Nacional (2003). 25 millones de dólares de financiamiento por parte del FONPLATA llegan en 2007. En el mar- 
co del proyecto para la reconversión del puerto de Santa Fe, un año más tarde, se autoriza la expropiación de bienes inmuebles necesarios para tal fin. En 2012, la provincia deja sin efecto la obligación del Ente Administrador del Puerto de Santa Fe de reintegrarle los desembolsos efectuados en el marco del convenio con el FONPLATA.

También se encontraron algunas iniciativas tendientes a normalizar el funcionamiento de cooperativas de trabajo creadas tras el cierre de empresas, declarando de utilidad pública los bienes y herramientas de dos industrias en 2009 y 2012, con posteriores ratificaciones.

Por otro lado, muy interesante fue la creación del Parque Tecnológico Litoral Centro SAPEM (2002) entre la provincia, el CONICET y la Universidad Nacional del Litoral, donde funciona una incubadora de empresas de base tecnológica que brinda servicios a emprendedores.

En otro orden de cosas, el Gobierno Provincial y la empresa Energía Argentina S.A. firmaron un convenio para la radicación de una generadora termoeléctrica en el Parque Industrial de Sauce Viejo, con el fin de procurar el crecimiento de la capacidad instalada en materia de generación de energía. Esta iniciativa fue clave para avanzar en la expansión de la actividad económica. Relacionado también con el Parque Industrial de Sauce Viejo, en 2011 se firma un convenio con la Asociación Civil Parque Industrial Sauce Viejo para reducir la contaminación ambiental ocasionada por la actividad industrial, mediante la elaboración de un plan para la gestión ambiental integral del sector industrial y la capacitación de recursos humanos.

Otras iniciativas fueron la sesión de terrenos a la Municipalidad de Recreo para la creación de un área industrial (2012) y a la Comuna de Arroyo Leyes para emprendimientos productivos (2006). También, el otorgamiento de asistencia financiera a distintos sectores productivos en 2004 y 2005.

\section{La costa}

La región de la costa es una de las menos pobladas y desarrolladas que, además, fue de las que menos atención recibió (después del noroeste). En el periodo, se transfirió el puerto a la localidad de Helvecia (1995); se realizó un censo hortícola (1996); se desarrolló un programa de asistencia técnica para la producción (2003) y se normalizó la situación de 
una industria recuperada (2005). El resto fueron leyes declarando de interés público actividades o lugares. Si bien se trata de una iniciativa que no fue instrumentada por ley, cabe destacar el plan "A toda costa", creado en 2016 por el decreto 4.200, destinado al desarrollo integral de los departamentos Garay y San Javier, que incluye, entre otras, desarrollo turístico, producción y trabajo, e infraestructura. A través del plan, se desarrollaron líneas de trabajo y programas ya existentes en el Ministerio de la Producción, y otras acciones que fueron producto del propio plan.

\section{Noreste}

En el noreste, el puerto de Reconquista corrió la misma suerte que los de Rosario, Santa Fe y Villa Constitución, conformándose en 1994 el Ente Administrador del Puerto Reconquista, luego de que el Estado Nacional lo transfiriera a la provincia. Años más tarde, en 1998, la Legislatura Provincial autoriza al Poder Ejecutivo a conceder una ayuda financiera al Ente de 150 mil pesos, para la reparación del acceso al pontón del atracadero de balsas y así reestablecer el servicio de transporte entre Reconquista y la vecina ciudad de Goya en la provincia de Corrientes.

También en materia de infraestructura, se autoriza al Poder Ejecutivo Provincial a endeudarse para el abastecimiento de agua en Reconquista, obra que, además, trasciende las finalidades productivas y es de aprovechamiento para el total de la población de la ciudad (2013).

Otras iniciativas relevantes fueron la creación de un parque industrial en la localidad de Avellaneda, en 2010, que junto a Reconquista conforman el mayor aglomerado del norte santafesino, y la creación del Polo Tecnológico Regional del Norte Santafesino, con sede en Reconquista, en 2018.

En 1998 y 2000 se aprueban leyes para la reconversión de la cuenca cañera y un programa para el desarrollo de la actividad forestal para el área noreste de Santa Fe. Estas iniciativas van en línea con la historia de la región, estrechamente ligada a la producción forestal.

Se encontró también medidas de normalización de la actividad de una fábrica recuperada en la ciudad de Vera $(2010$, con sucesivas prórrogas) y la donación de terrenos a pequeños productores aborígenes, también de esa ciudad (2002). 
Sin embargo, si bien no se trata de una ley, tal vez la línea de trabajo más importante que se haya articulado para esta región sea el Plan del Norte, instrumentado a partir del año 2016. La iniciativa se basa en el decreto provincial $0015 / 2016$ y existe un proyecto de ley para darle continuidad. El plan contempla el desarrollo integral de los departamentos 9 de julio, Vera y General Obligado a través de tres ejes. El tercero, denominado "Economía para el desarrollo", contiene líneas de trabajo en infraestructura y energía, investigación e innovación tecnológica, asociativismo y comercialización, economías regionales y agregado de valor y desarrollo industrial. A través del plan se desarrollaron líneas de trabajo y programas ya existentes en el Ministerio de la Producción, $\mathrm{y}$ otras acciones que fueron producto del propio plan.

\section{Noroeste}

La región noroeste recibió solo dos iniciativas directas: la autorización por parte de la Legislatura al Ejecutivo para contraer deudas de 16 y 36 millones de dólares, con el fin de mejorar caminos rurales en los años 1996 y 1998 respectivamente. A esto hay que sumar los proyectos articulados a través del recientemente mencionado Plan del Norte.

\section{Cuenca lechera}

En 1997, se aprueba un convenio para la constitución del "Centro de Innovación Tecnológica Rafaela”, el cual, en el año 2000, se transformó en un centro regional del INTI. También en Rafaela (1994) se aprueba un convenio entre el Gobierno Provincial, el Gobierno Nacional y el Centro Comercial e Industrial del Departamento Castellanos y sus empresas asociadas, para la reconversión y crecimiento de un polo productivo. Iniciativas que avanzan también en esta línea son las habilitaciones para expropiar terrenos para la constitución de un parque tecnológico en las ciudades de San Jorge (2007) y Gálvez (2013). También en este sentido, en 2018 se avanzó con la creación de un polo tecnológico en la ciudad de Esperanza.

Otras acciones fueron un convenio entre el Ministerio de Agricultura, Ganadería, Industria y Comercio con la Fundación las Colonias (1997); un plan de entrenamiento para la producción de miel, conforme a protocolos de calidad (2000), en el departamento San Martín, en el centro-oeste de la provincia; expropiación y uso temporal 
de maquinarias de una fábrica recuperada en Cañada de Rosquín (2006), y una línea de créditos especiales para damnificados por un tornado en 2004.

\section{Pampeana}

En 1994 se firmó un convenio entre el Estado Nacional, la provincia y el Centro Industrial de las Parejas (al que adhirieron cinco municipios $\mathrm{y}$ tres comunas del suroeste santafesino), destinado al fortalecimiento de la maquinaria agrícola e industrias afines. También en Las Parejas se habilitó al municipio para contraer deuda por 350 mil dólares para ampliar su parque industrial (1997). Pueblo Andino (2003), Serodino (2006) Bigand (2007) y Cañada de Gómez (2004 y 2014) fueron beneficiados por iniciativas relacionadas con la sesión de terrenos para zonas industriales.

Interesante también resulta la sanción del "Área de planificación estratégica ambiental del humedal de la Laguna de Melincué" (1998), con fines ecológicos pero también turísticos.

Otro tipo de leyes relacionadas con la producción del área en cuestión tiene que ver con bonificaciones de tasas en una línea de créditos habilitada por el CFI para la producción agropecuaria, industrial y de servicios afectados por fenómenos meteorológicos (2007); y ayuda económica a productores rurales afectados por el crecimiento de la Laguna La Picasa, en el extremo suroeste de la provincia (2008).

También, se encontró legislación que declara de utilidad pública los bienes de empresas recuperadas, tal como se describió; y reconocimientos a localidades como "Capital Provincial de...."

A continuación, en la tabla 2, se muestra la frecuencia para cada una de las áreas trabajadas, de acuerdo con la categorización mencionada más arriba. 
Tabla $\mathrm{N}^{\circ} 2$

Clasificación de las menciones a temáticas específicas en las leyes sancionadas por la Legislatura agregadas por áreas. Provincia de Santa Fe, 1991-2019

\begin{tabular}{|c|c|c|c|c|c|c|c|c|c|c|c|c|}
\hline & 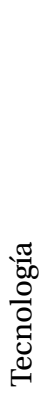 & 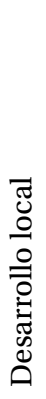 & 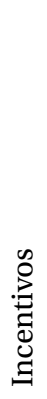 & 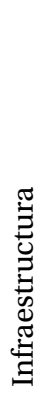 & 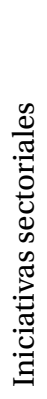 & 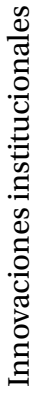 & 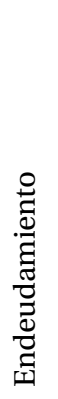 & 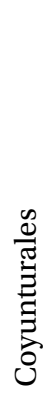 & 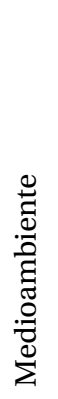 & 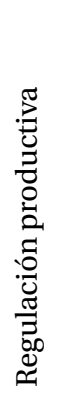 & 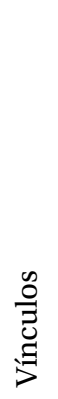 & 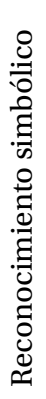 \\
\hline Área geográfica & \multicolumn{12}{|c|}{ Menciones } \\
\hline Cordón industrial & 1 & 6 & 2 & 8 & 12 & 6 & 0 & 11 & 1 & 0 & 13 & 4 \\
\hline Gran Rosario & 1 & 5 & 1 & 5 & 12 & 1 & 0 & 11 & 1 & 0 & 11 & 2 \\
\hline Pampeana & 0 & 4 & 2 & 0 & 8 & 1 & 1 & 2 & 1 & 0 & 5 & 7 \\
\hline Gran Santa Fe & 2 & 3 & 3 & 8 & 4 & 1 & 2 & 9 & 1 & 1 & 9 & 0 \\
\hline Cuenca lechera & 2 & 6 & 2 & 0 & 7 & 0 & 0 & 2 & 0 & 0 & 6 & 1 \\
\hline Noreste & 1 & 3 & 0 & 2 & 7 & 1 & 1 & 6 & 0 & 0 & 5 & 2 \\
\hline $\begin{array}{l}\text { Reconquista- } \\
\text { Avellaneda }\end{array}$ & 1 & 0 & 0 & 2 & 1 & 1 & 0 & 1 & 0 & 0 & 0 & 2 \\
\hline La Costa & 0 & 1 & 0 & 3 & 2 & 0 & 0 & 2 & 0 & 0 & 2 & 4 \\
\hline Noroeste & 0 & 1 & 0 & 1 & 2 & 0 & 1 & 0 & 0 & 0 & 1 & 0 \\
\hline
\end{tabular}

Fuente: Elaboración propia.

\section{Comparación temporal}

En el ámbito nacional, los 28 años transcurridos entre 1991 y 2019 permiten identificar cinco etapas: la etapa aperturista y de reforma del Estado durante la década de los 90; el periodo de crisis con epicentro en el 19 y 20 de diciembre de 2001; la reactivación de la economía con altas tasas de crecimiento entre 2003 y 2007; una etapa de crecimiento leve y contracciones leves desde entonces hasta 2015, y el periodo de recesión económica, desindustrialización, alta inflación, endeudamiento y devaluación del gobierno de Macri, desde 2016 hasta 2019. Estos cinco periodos son más o menos coincidentes, en el nivel provincial, con diferentes gobernaciones. La primera etapa 
con el primer periodo de gobierno de Carlos Reutemann (1991-1995) y la primera gobernación de Jorge Obeid (1995- 1999); la etapa de recesión y crisis con el segundo periodo de Reutemann (1999-2003); el crecimiento a altas tasas, con el segundo de Obeid (2003-2007); el cuarto con los mandatos Hermes Binner (2007-2011) y Antonio Bonfatti (2011-2015); y el último con Miguel Lifschitz (2015-2019). Esta situación permitió esbozar una comparación coincidente entre los cambios macroeconómicos que afectaron al país y a la provincia, y los sucesivos gobiernos provinciales.

En líneas generales, resultó muy difícil establecer regularidades distintivas de cada periodo. Lo mismo ocurrió a la hora de intentar encontrar líneas de acción que hayan atravesado la totalidad de las administraciones provinciales mencionadas; aunque existen algunas excepciones, como la política de parques industriales, que se mantuvo relativamente constante durante los 28 años analizados, y las acciones de apoyo a las empresas recuperadas, que desde 2002 en adelante fueron sostenidas en todos los periodos mencionados. Sobre el resto, se cuentan medidas aisladas o discontinuadas que se articularon de manera reactiva frente a los cambios del contexto, o que debieron ser abandonadas debido también a la inestabilidad macro.

El primer dato que llama la atención es que las zonas menos desarrolladas fueron, también, las que menos atención recibieron durante todos los años de la serie (por lo menos en lo que respecta a legislación). Si bien la región noreste recibió una atención relativamente alta en comparación con la costa y el noroeste, se ve también que casi la mitad de las iniciativas fue dirigida hacia la mayor y más desarrollada área urbana de la región, conformada por las vecinas ciudades de Reconquista y Avellaneda. Una situación que se propuso remediar la articulación del "Plan del Norte" y del plan "A Toda Costa", a partir de 2016.

Se observa, a su vez, que la zona industrial del sureste recibió mayor atención en todos los periodos, excepto durante el segundo gobierno de Reutemann y el periodo de Binner al frente de la gobernación provincial. En esta última administración, el área con mayor número de menciones en los documentos analizados fue el Gran Santa Fe, básicamente por cuestiones relacionadas con el puerto de la ciudad capital. El área pampeana y la cuenca lechera recibieron relativamente 
baja atención en casi todos los periodos, excepto en el segundo de Obeid, que se caracterizó por una mayor actividad que acompañó el proceso de reactivación económica del país. Durante esta etapa se encontró la mayor cantidad de iniciativas dirigidas a la industria y a las producciones primarias alternativas.

Durante la crisis del 2001 y sus años posteriores, la provincia sancionó una cantidad de iniciativas considerables a favor de la industria láctea, cuya actividad se ubica mayormente en el centro oeste santafesino. Durante esos años también se encontró el mayor número de iniciativas hacia la actividad ictícola, que favorecieron a la zona de la costa.

Los periodos comprendidos entre 1996-1998 y 2003-2007 tienen en común ser situaciones de reactivación económica. Ambas etapas corresponden a las dos gobernaciones de Obeid. Se puede destacar como diferencia que durante la primera gobernación de Obeid se vieron más iniciativas de asistencia técnica, mientras que durante su segundo periodo al frente del gobierno provincial se encontraron más iniciativas de apoyo financiero a la producción.

Las iniciativas vinculadas con municipios y comunas fueron más frecuentes durante los años 90, básicamente a causa del traspaso de puertos ubicados en ciudades costeras del Paraná a la órbita municipal.

En la Tabla Nº3 se muestran los datos para cada período de gobierno: 
Tabla $\mathrm{N}^{\circ} 3$

Menciones a localidades y departamentos en las leyes sancionadas por la Legislatura agregados por zona y según periodo de gobierno. Provincia de Santa Fe, 1991- 2019

\begin{tabular}{|c|c|c|c|c|c|c|c|c|c|c|}
\hline & 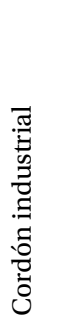 & 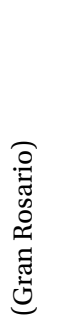 & 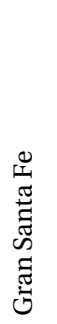 & 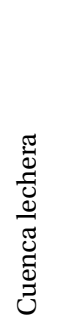 & 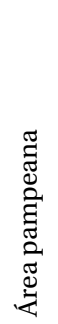 & $\begin{array}{l}\stackrel{0}{0} \\
\stackrel{0}{0} \\
\vdots \\
z\end{array}$ & 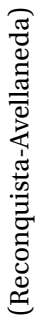 & 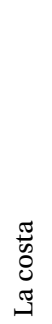 & $\begin{array}{l}0 \\
\stackrel{0}{0} \\
0 \\
0 \\
z\end{array}$ & స్త్ర \\
\hline Periodo & \multicolumn{10}{|c|}{ Menciones } \\
\hline Reutemann (1991-1995) & 6 & 3 & 1 & 3 & 3 & 2 & 2 & 4 & 0 & 19 \\
\hline Obeid (1995- 1999) & 7 & 7 & 3 & 1 & 2 & 1 & 1 & 2 & 2 & 18 \\
\hline Reutemann (1999-2003) & 5 & 2 & 3 & 4 & 2 & 6 & 1 & 1 & 0 & 21 \\
\hline Obeid (2003- 2007) & 11 & 10 & 3 & 7 & 8 & 0 & 0 & 3 & 0 & 32 \\
\hline Binner (2007- 2011) & 4 & 4 & 8 & 2 & 3 & 3 & 2 & 1 & 0 & 21 \\
\hline Bonfatti (2011- 2015) & 6 & 3 & 3 & 3 & 1 & 2 & 0 & 0 & 0 & 15 \\
\hline Lifschitz (2015-2019) & 3 & 2 & 4 & 3 & 1 & 4 & 2 & 0 & 0 & 15 \\
\hline Total & 42 & 31 & 25 & 23 & 19 & 18 & 8 & 11 & 2 & \\
\hline
\end{tabular}

Fuente: Elaboración propia.

\section{Consideraciones finales}

Para lograr disminuir los desequilibrios entre regiones debe llevarse adelante políticas de desarrollo que consideren al territorio en su conjunto. En este sentido, no existió en ninguno de los periodos analizados una política clara para la disminución de la brecha norte-sur en Santa Fe, excepto los planes del norte y de la costa implementados por el gobierno de Lifschitz, los cuales no lograron ser sancionados como ley por la Legislatura provincial, pero sí avanzaron con algunas acciones. Además, cabe destacar que, salvo por la política de parques industriales, tampoco hubo iniciativas que se hayan mantenido activas durante el total de los años analizados. Las políticas productivas del Estado provincial se vieron afectadas por la inestabilidad del contexto nacional que, las más de las veces, impactó de forma negativa sobre la economía santafesina. En este sentido, se observó que la provincia 
cuenta con una capacidad relativamente importante de reacción frente a los efectos de coyunturas económicas desfavorables - la cual se ve potenciada cuando opera en consonancia con el Estado nacional-, pero es mucho más limitada su posibilidad de desplegar una política de desarrollo económico relativamente autónoma.

Se vio cómo la situación de la economía nacional y las reformas llevadas adelante por el gobierno central durante la década del 90 fueron factores que impactaron directamente sobre la vida económica provincial. Así, el Estado provincial, a la vez que operó como contraparte del Estado nacional en el proceso de transferencia de funciones, tuvo también que hacer frente a la situación económica generada por el contexto de desregulación y apertura de la economía. En ese contexto, las iniciativas articuladas desde la provincia avanzaron intentando mitigar el impacto negativo de las políticas nacionales y otro tipo de contingencias.

Por otro lado, vimos que fue particularmente significativo el rol del Estado provincial en los años posteriores a la salida de la crisis de 2001, que fue cuando se encontró el mayor número de iniciativas del periodo analizado. Allí observamos cómo operó, acompañando y ayudando a apuntalar la reactivación económica. Como dijimos, esto indica que, al menos en Santa Fe, el Estado provincial tiene la capacidad de jugar un rol activo en momentos en que es necesario ayudar a la solución de problemas, pero no la capacidad de anticiparlos. Las más de las veces se trató de un comportamiento reactivo, cuya intención fue mitigar las consecuencias negativas generadas por las recurrentes crisis de la economía nacional. Así se vio cómo, incluso en periodos de crecimiento económico, existieron dificultades para articular acciones estratégicas destinadas a corregir los históricos desequilibrios territoriales internos. Esta situación indica la necesidad de indagar, con mayor profundidad, acerca del rol y las posibilidades de los Estados provinciales en Argentina de funcionar como agentes dinamizadores del desarrollo económico.

Por otro lado, vimos también cómo, durante la reactivación económica después de 2003, las zonas más desarrolladas —en ese entonces deprimidas por la crisis económica desatada en el 2001 - fueron las que lograron captar en mayor medida la atención del Estado provincial. No es de extrañar el caso del Gran Rosario, ya que se trata de la 
región más poblada y la que mayor sufrió el modelo económico aperturista de los años 90; pero sí es particularmente significativo el caso del sector lácteo, el cual fue beneficiario de una importante cantidad de ayudas económicas directas e indirectas. Esto hace surgir la interrogante acerca de la capacidad de llegada de los diferentes sectores al Estado provincial; pero también respecto de la posibilidad de influencia de algunos actores económicos ubicados en zonas geográficas determinadas. En este sentido, sería interesante, en el futuro, indagar con mayor detalle respecto de la articulación entre política y economía en el proceso de construcción territorial de poder al interior de la provincia, focalizando en lobbys sectoriales, pero también en actores territoriales.

También sería importante analizar el rol de los actores políticos de las regiones económicamente más deprimidas, quienes, quedó claro, ejercen una influencia muy limitada sobre la posibilidad de desarrollo económico de sus regiones; pero, sin embargo, dada la arquitectura institucional del poder legislativo santafesino, tienen una gravitación política relativamente amplia sobre las decisiones del Estado provincial. En este sentido, indagar sobre la agenda política de los legisladores del norte provincial puede ser una línea de investigación relevante de cara al futuro.

En líneas generales, a modo de cierre, se puede decir que, en el caso santafesino, se observaron las dificultades a las que se enfrentan las regiones de la periferia o la semiperiferia para articular un modelo de desarrollo que pueda hacer frente a las dinámicas de la economía global, incluso en áreas que internamente se encuentran en una situación relativa favorable. Las iniciativas analizadas dejan ver también el déficit de capacidades institucionales de las instancias de gobierno provinciales, para procesar y dar una respuesta efectiva a los desafíos generados por las transformaciones de su entorno.

De cara al futuro, el Estado provincial tiene por delante la tarea de incrementar sus capacidades estatales para gestionar los desequilibrios territoriales, pero sin perder de vista que las áreas con un desarrollo relativo mayor también deben consolidarse. En este sentido, debe trabajar en miras de potenciar las capacidades existentes en las regiones con mayor avance relativo y que este desarrollo, a su vez, pueda funcionar como un estímulo sobre las zonas económicamente de- 
primidas. El desafío mayor es lograr proyectar acciones estratégicas a largo plazo, que logren desplegar acciones sostenidas en el tiempo y anticiparse a los problemas coyunturales y a las contingencias. En este sentido, es clave la acción coordinada con los actores locales, estimulando el fortalecimiento de estos últimos para que puedan funcionar, también, como agentes promotores del desarrollo de su región.

\section{Referencias bibliográficas}

a) Artículos, capítulos de libros y presentaciones en congresos

Alburquerque, F. y Pérez Rozzi S. (2013). "El desarrollo territorial: enfoque, contenidos y política", en Revista Iberoamericana de Gobierno Local, ํㅜ 4, pp. 157-171.

Alonso, G. (2007). "Las capacidades institucionales entre el Estado y la sociedad". Presentación al $4^{\circ}$ Congreso Argentino de Administración Pública. Argentina: Buenos Aires.

Amin, A. (1998). "Una perspectiva institucionalista sobre el desarrollo económico regional", en Ekonomiaz. Revista Vasca de Economía, № 41, pp. 68-89.

Beckert, J. (2009). “El orden social de los mercados", en Comunicación, cultura y política. Revista de Ciencias Sociales, vol. 1, N² pp. 147-172.

Bertranou, J. (2013). "Capacidad estatal: aportes al debate conceptual". Presentación al $7^{\circ}$ Congreso Argentino de Administración Pública. Argentina: Mendoza, pp. 18-20.

Boisier, S. (2001). "Desarrollo local: ¿de qué estamos hablando?”, en O. Madoery y A. Vázquez Barquero (eds.) Transformaciones globales, Instituciones y Politicas de desarrollo local. Rosario: Homo Sapiens, pp. 48-74.

Fernández V. R. y J. Vigil. (2012). “Capacidades estatales regionales: consideraciones teóricas y metodológicas para su análisis en América Latina", en Revista Políticas Públicas, vol. 16, $\mathrm{N}^{\circ}$ 1, pp. 51-65.

FernándezV.R.yJ.Vigil.(2010). "ElEstado al primer plano del desarrollo (regional) latinoamericano. Un estudio de las capacidades estatales al nivel meso-regional en la Argentina", en Revista Documentos y Aportes en Administración Pública y Gestión Estatal, $\mathrm{N}^{\circ} 15$, pp. 43-90. 
Granovetter, M. y McGuire, P. (1998). "The making of an industry: electricity in the United States", en M. Callon (ed.), The Laws of Markets. Oxford: Blackwell, pp. 147-173.

Granovetter, M. (1973). "The strength of weak ties", en American Journal of Sociology, vol. 78, $\mathrm{N}^{\circ}$ 6, pp. 1360-1380.

Lattuada, M. y Nogueira, M. E. (2011). "Capacidades estatales y políticas públicas. Una propuesta para el abordaje de las políticas agropecuarias en la Argentina contemporánea (1991-2011)", en Estudios Rurales. Publicación de Centro de Estudios de La Argentina Rural, vol. 1, № 1, pp. 30-54.

Orlansky, D. (2005) "El Concepto de Desarrollo y las Reformas Estatales: Visiones de los Noventa", en Revista Documentos y Aportes en Administración Pública y Gestión Estatal, $\mathrm{N}^{\circ}$ 6, pp. 41-61.

Ortiz de Rozas, V. (2016). "Los estudios sobre política subnacional en Argentina: un recorrido por diferentes disciplinas y perspectivas. Sobre los aportes de una escala de análisis y su afinidad con un enfoque centrado en los actores políticos y sus prácticas", en Cuadernos FHyCS-UNJu, $\mathrm{N}^{\circ}$ 50, pp. 57-80.

Vilas, C. (2005). “ ¿Estado víctima o Estado promotor? El debate sobre soberanía y autonomía en el capitalismo globalizado", en C. M. Vilas, Estado y política en la Argentina actual. Buenos Aires: Universidad Nacional de General Sarmiento/ Prometeo, pp. 21-65.

\section{b) Libros}

Albuquerque, F. (2004). El enfoque del desarrollo económico local. Buenos Aires: OIT.

Barros, S., A. Castellani y D. Gantus (2016) (coord.). Estudios sobre Estado, gobierno y administración pública en la Argentina contemporánea. Buenos Aires: CODESOC, PISAC y CLACSO.

Glaser, B. y A. Strauss (1967). The discovery of grounded theory: strategies for qualitative research. Nueva York: Aldine Publishing Company.

Mauro, S., V. Ortiz de Rozas y P. Vaca Narvaja (2016) (comp). Política subnacional en Argentina. Enfoques y problemas. Buenos Aires: CEAP, UBA.

Polanyi, K. (2007). La gran transformación. Madrid: Ediciones de la Piqueta. 
Vázquez Barquero, A. (2000). Desarrollo económico local y descentralización: aproximación a un marco conceptual. Santiago de Chile: CEPAL.

c) Documentos de trabajo

Acosta, S. (2011a). "Cadena de valor autopartista". Observatorio Pyme Regional Provincia de Santa Fe. Buenos Aires: Fundación Observatorio Pyme y Ministerio de la Producción de la Provincia de Santa Fe.

Acosta, S. (2011b). "Cadena de valor de la carne bovina". Observatorio Pyme Regional Provincia de Santa Fe. Buenos Aires: Fundación Observatorio Pyme y Ministerio de la Producción de la Provincia de Santa Fe.

Acosta, S. (2011c). "Cadena de valor carrocera, remolques y semirremolques". Observatorio Pyme Regional Provincia de Santa $\mathrm{Fe}$. Buenos Aires: Fundación Observatorio Pyme y Ministerio de la Producción de la Provincia de Santa Fe.

Acosta, S. (2011d). "Cadena de valor láctea”. Observatorio Pyme Regional Provincia de Santa Fe. Buenos Aires: Fundación Observatorio Pyme y Ministerio de la Producción de la Provincia de Santa Fe.

Acosta, S. (2011e). “Cadena de valor de madera y muebles". Observatorio Pyme Regional Provincia de Santa Fe. Buenos Aires: Fundación Observatorio Pyme y Ministerio de la Producción de la Provincia de Santa Fe.

Acosta, S. (2012a). "Cadena de valor de la maquinaria para la industria alimenticia". Observatorio Pyme Regional Provincia de Santa $\mathrm{Fe}$. Buenos Aires: Fundación Observatorio Pyme y Ministerio de la Producción de la Provincia de Santa Fe.

Acosta, S. (2012b). "Cadena de valor textil y confecciones". Observatorio Pyme Regional Provincia de Santa Fe. Buenos Aires: Fundación Observatorio Pyme y Ministerio de la Producción de la Provincia de Santa Fe.

De Mattos, C. A. (2012). “De la planificación a la gobernanza. Hacia un nuevo modo de gestión urbana". Santiago: IEUT/UC.

Donato, V. (2010). “Industria manufacturera año 2009”. Observatorio Pyme Regional provincia de Santa Fe. Buenos Aires: Fundación Observatorio Pyme y Ministerio de la Producción de la Provincia de Santa Fe. 
Repetto F. (2004). "Capacidad estatal: requisito para el mejoramiento de la política estatal en América Latina". Documentos de Trabajo del INDES I- 52.

d) Otros

Dirección Nacional de Relaciones Económicas con las Provincias. Recuperado de http://www2.mecon.gov.ar/hacienda/dinrep/ competencias/dinrep.php

Instituto Nacional de Estadística y Censos (INDEC). Recuperado de https://www.indec.gob.ar/

Sistema de información normativa de la Provincia de Santa Fe Recuperado de https://www.santafe.gov.ar/normativa/ 